\title{
Personalising and targeting antiangiogenic resistance: a complex and multifactorial approach
}

\author{
Maria J Bueno ${ }^{1,3}$, Silvana Mouron ${ }^{1,3}$ and Miguel Quintela-Fandino *,1,2 \\ ${ }^{1}$ Breast Cancer Clinical Research Unit, CNIO_Spanish National Research Cancer Centre, Madrid, Spain and ${ }^{2}$ Medical Oncology, \\ Hospital Universitario Quirón, Pozuelo de Alarcón, Madrid, Spain
}

Pathological angiogenesis involves complex and dynamic interactions between tumour cells and other lineages existing in the microenvironment of the tumour. Preclinical and clinical data suggest that tumours can show dual, different adaptive responses against antiangiogenic agents: one successful adaptation is vascular normalisation, whereas the second adaptation is elicited through vascular trimming and increased hypoxia. These phenomena depend on the type of tumour and the type of agent. The classical approach for investigating acquired resistance against antiangiogenic agents is to identify compensatory signalling pathways emerging in response to VEGF blockade, which has led to the development of highly effective drugs; however, ultimately these drugs fail. Here we review how the dual stromal adaptive patterns determine the mechanisms of escape that go beyond the reprogramming of signal transduction pathways, which obliges us to investigate the tumour as an ecosystem and to develop uni- and multicompartmental models that explain drug resistance involving metabolic and immune reprogramming. We also propose a method for facilitating personalised therapeutic decisions, which uses 18F-fluoromisonidazole-positron emission tomography to monitor the dual stromal response in tumours of individual patients.

Under physiological conditions, angiogenesis is tightly regulated, and leads to a mature, well-constructed vascular network. By contrast, the normal regulation of angiogenesis fails under pathologic conditions such as cancer, and aberrant formation of blood vessels frequently occurs. This structurally abnormal network leads to aberrant local blood flow, fluid dynamics, and oxygenation, resulting in tumour growth and local and metastatic invasion by cancer cells (Jain, 2013). Research on the understanding of the process of angiogenesis has evolved considerably since Judah Folkman first introduced the hypothesis of the 'diffusion limit of oxygen' (Folkman, 1971). Considering the essential role of angiogenesis in tumour growth and progression, Folkman proposed the targeting of angiogenesis signalling molecules as a new approach for treating human cancer (Folkman, 1990). Since then, considerable effort has gone into the development of antiangiogenic drugs, and a number of these inhibitory agents have been approved by the FDA for clinical use against several types of cancer. However, tumours often escape the effects of these agents, and the disease eventually progresses. Elucidating the molecular mechanisms underlying the adaptive tumour response to antiangiogenic agents is a major need in clinical oncology.

\section{RESISTANCE AGAINST ANTIANGIOGENIC AGENTS: A CLASSIC SIGNALLING PERSPECTIVE}

Traditionally, the study of antiangiogenic resistance has focused on the vascular endothelial growth factor (VEGF) axis, including the proangiogenic driver VEGF-A and its receptor VEGFR-2. The development of antiangiogenic drugs has focused for decades on the inhibition of VEGF. The first agent receiving FDA approval was bevacizumab, a humanised monoclonal antibody against VEGF-A; bevacizumab administered in combination with chemotherapy increased the overall survival of colorectal cancer patients by 5 months (Hurwitz et al, 2004). Multitargeted tyrosine kinase inhibitors (TKIs) target the kinase domains of the tyrosine kinase 
VEGF receptors and other tyrosine kinase receptors. Among the TKIs, sorafenib and sunitinib have been tested most extensively in phase III trials, showing positive results in liver and kidney cancers. The failure of this therapeutic approach due to acquired resistance has led to the increased understanding of VEGF-independent angiogenesis. Chronic VEGF/VEGFR2 inhibition was found to lead to the emergence of specific compensatory signalling pathways that sustain angiogenesis. These pathways rely on key proteins, including fibroblast growth factor (FGF) and its receptors (FGFR; Pepper et al, 1992), angiopoietin-2 (Ang2; Oliner et al, 2004), and the MET oncogene (Lu et al, 2012). These signalling pathways have been shown to regulate angiogenesis and serve as alternative inducers of tumour growth. Over past decades, complementary therapies that inhibit angiogenesis indirectly have been developed in attempts to abrogate acquired resistance. Targeting the Ang-Tie axis was proven successful, at least for ovarian cancer, where trebananib, a peptibody that inhibits the binding of Ang1/2 to their receptor, Tie2, was granted FDA approval in lieu of a phase III trial (Monk et al, 2014). Similarly, cabozantinib, an anti-MET agent, was successful in a phase III trial of patients with kidney cancer who had been previously exposed to anti-VEGFR2 therapy (Choueiri et al, 2015). These results support the theory that these axes are involved in compensatory responses against chronic VEGFR2 blockade. Unfortunately, despite these new approaches, treatment failure ultimately occurs in every patient with metastatic cancer. The obvious conclusions are that different mechanisms account for the perpetuation of pathologic angiogenesis, and that acquired resistance requires other approaches beyond the inhibition of signal transduction. Evidence increasingly points to tumour microenvironment and interactions between tumour cells and non-malignant cells as factors involved in the adaptive response of cancers against antiangiogenic agents. Thus, the mechanism of resistance to antiangiogenic therapies must be thought of as a dynamic multifactorial process. Integrating the inhibition of signalling pathways with the manipulation of the dynamic changes in tumour microenvironment and stromal cell interactions may optimise the inhibition of angiogenesis and overcome resistance, therefore improving patient outcomes (Figure 1).

DUAL PATTERN OF MICROENVIRONMENTAL RESPONSE AND RESISTANCE AGAINST ANTIANGIOGENIC THERAPIES

For years, 'vascular normalisation' has been assumed to be the general mechanism of action of antiangiogenic agents (Jain, 2013). The 'normality' concept applies not only to the blood vessels and their structure but also to the intercellular matrix, the type and polarisation of leukocytes, and fibroblasts (Huang et al, 2012; Jain, 2013). However, sufficient available preclinical and clinical data strongly indicate that antiangiogenic treatment is not always followed by vascular normalisation, and that tumours can manifest dual, different adaptive responses against antiangiogenic agents. One successful adaptation is vascular normalisation (followed by hypoxia correction), whereas the second adaptation is elicited through vascular pruning followed by increased hypoxia (Sennino and McDonald, 2012). The type of response would be determined by the type of agent, dynamic changes in the concentrations of proand antiangiogenic factors within the tumour microenvironment (and thus the timing of treatment administrations), and tumour type. The heterogeneity of adaptive responses to antiangiogenic therapy has implications for tumour metabolic states and the distribution of infiltrating immune cells infiltrate, which, in turn, impact the mechanisms of acquired resistance (Figure 2).

One study of patients with lung cancer who received radiolabelled docetaxel and underwent PET found that bevacizumab reduced both the perfusion and the net influx rate of docetaxel into the tumour, which contradicts the theoretical effects of normalisation (Van der Veldt et al, 2012). Similar controversial results have been found for sunitinib and sorafenib. Using electron paramagnetic resonance imaging of mice with squamous cell carcinoma xenografts, Matsumoto et al (2011) found that sunitinib normalised vasculature and tumour oxygenation. However, other investigators have found that sunitinib increased hypoxia, and that this effect was associated with epithelial-to-mesenchymal transition, stimulation of cancer stem cells, and metastases (Cooke et al, 2012). Similarly, results of other studies have shown this dual response to sorafenib (Murphy et al, 2006; Kumar et al, 2007; Liu et al, 2012). Taken together, these data suggest that responses to antiangiogenics include either decreased or increased tumour hypoxia, and that these responses might be tumour-dependent and agent-dependent. We sought to clarify these suppositions. In one experiment, we explored the 'tumour variable' and found that the same TKI (dovitinib) administered to two different pancreatic cancer tumourgraft models induced vascular and hypoxia normalisation in one model (followed by improved delivery of interstitial chemotherapy), while the opposite effects were observed in the other one (Hernandez-Agudo et al, 2016). In a second experiment, we explored the 'agent variable' and tested several multi-TKIs (nintedanib, dovitinib, and axitinib) and a monoclonal antibody (B20-4.1.1) on the same tumour model (MMTV-PyMT). We found that both axitinib and B20-4.1.1 increased hypoxia, while the other agents corrected it (Navarro et al, 2016). Given the unpredictable responses (whether or not an antiangiogenic agent will normalise a specific tumour), we realised that identifying which of the two possible stromal responses was occurring in an individual patient was highly relevant. Accordingly, we demonstrated that the vascular normalisation response could be monitored by a non-invasive test (PET using 18F-fluoromisonidazole, a probe that binds to tissues with $<1 \%$ oxygen) (Hernandez-Agudo et al, 2016). Interestingly, these findings were validated in the clinical setting, where $18 \mathrm{~F}$-fluoromisonidazolepositron emission tomography (18F-MISO-PET) demonstrated that patients undergoing neoadjuvant treatment with nintedanib could show both increases or normalisation of hypoxia, and that the pattern of hypoxia correction was correlated with response (Quintela-Fandino et al, 2016).

ANTIANGIOGENIC RESISTANCE IN THE CONTEXT OF NORMALISATION: UNICOMPARTMENTAL MODEL

We recently explored the mechanism of adaptation against the novel antiangiogenic TKIs, nintedanib and dovitinib. The key finding was that the agents that acted to correct the abnormal physiology of tumour blood vessels and to correct hypoxia ultimately led to a major switch in cancer metabolism (Navarro et al, 2016). The high glucose uptake that is inherent to most epithelial malignancies (especially in MAPK- and/or Pi3K-AKTactivated tumours), which is also known as the Warburg effect, is needed not only to satisfy energy requirements but also to supply additional carbon skeletons required by an anaplerotic shift in the Krebs cycle (Lunt and Vander Heiden, 2011). Our data showed that although glycolysis was restricted in the presence of antiangiogenic TKIs, because of changes in HIF1- $\alpha$ and AMPK signalling, the tumours continued growing normally. This finding suggests that under selective pressure, tumour plasticity led to sustained tumour growth over the long term. However, under these conditions, the tumours were dependent on the continuous uptake and degradation of fatty acids and ketone bodies, which are targeted for mitochondrial catabolism. This switch to an alternative metabolic source became essential for tumour survival. When several breast and lung cancer models were 'primed' with normalising antiangiogenic TKIs, the use of mitochondrial 


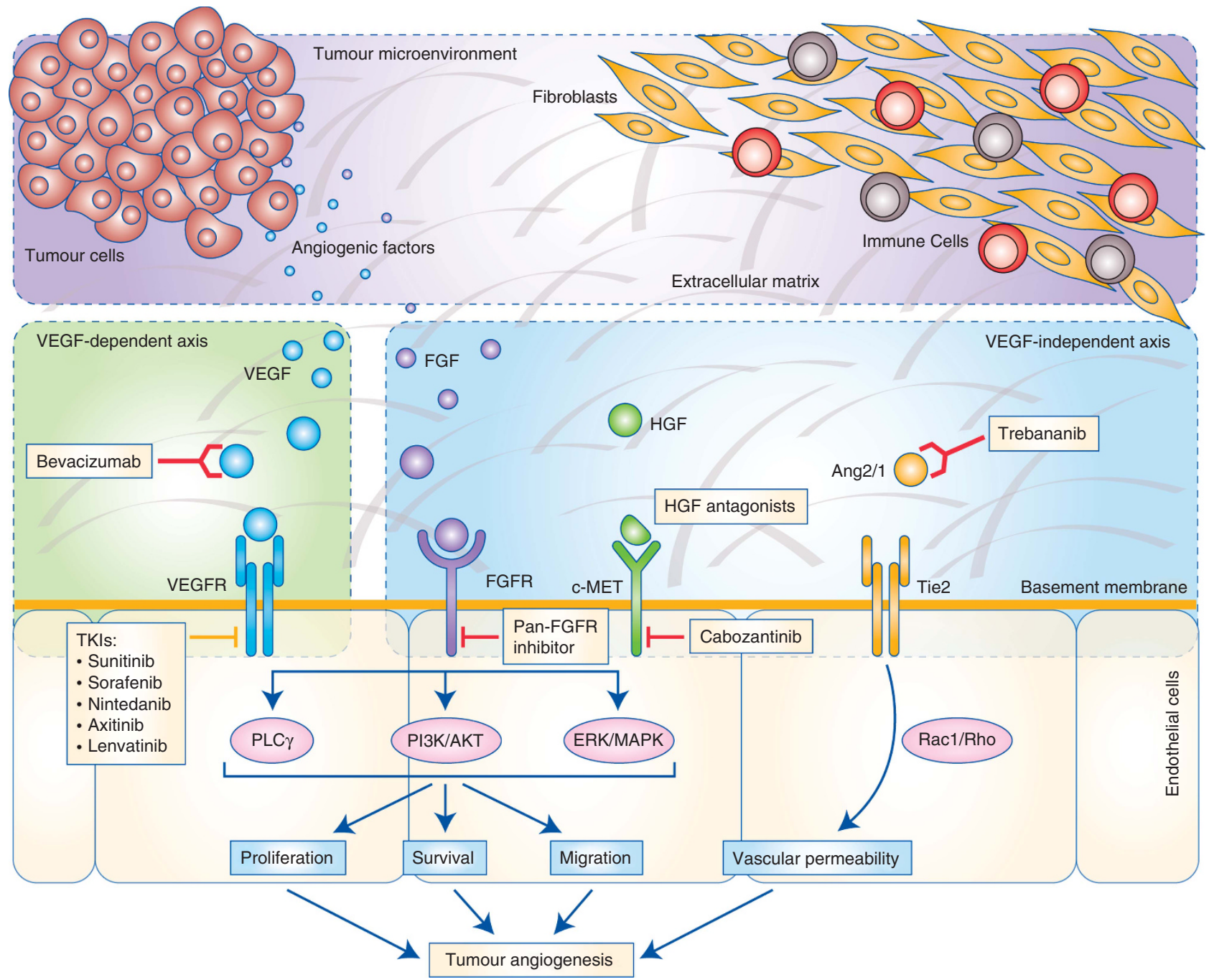

Figure 1. Tumour angiogenesis: molecular pathways and potential interventional strategies. VEGF axis is recognised as the primary factor responsible for tumour angiogenesis resistance. Upon VEGF binding, the receptor tyrosine is activated. VEGF/VEGFR activates oncogenic signalling via mitogen-activated protein kinase (ERK/MAPK) pathway, the phosphotidylinositol 3 kinase (PI3K-AKT) pathway, and the phospholipase-C- $\gamma$ (PLC- $\gamma$ ) pathway leading to cell proliferation, survival, migration, and vascular permeability. Chronic VEGF/VEGFR inhibition has been found to lead to the emergence of compensatory signalling pathways that sustain angiogenesis. Several of these VEGF-independent signalling pathways, rely on key proteins, including the FGF/FGFR, angiopoietin-2 (Ang2), and the MET oncogene. Complementary therapies focused on these alternative pathways have been developed to abrogate acquired resistance. Pan-FGFR inhibitors are used to inhibit the FGF/ FGFR axis. The MET/HGF pathway is inhibited by HGF antagonists and by the anti-MET antibody cabozantinib. Ang-Tie axis can be targeted with trebananib, a peptibody that inhibits the binding of Ang1/2 to their receptor. Tumour microenvironment and interactions between tumour cells and non-malignant cells as fibroblast and leukocytes also affect angiogenesis by interfering with the signalling pathways required for cell recruitment and vascular construction.

inhibitors (phenformin or ME-344) led to impressive antitumour effects. The dependence on mitochondrial metabolism was only evident in this situation, as the antimitochondrial agents were inactive when used in monotherapy. This point is of clinical relevance because of the rising interest in biguanides in the medical community. Biguanides have been associated to lower cancer burden in diabetic patients (Evans et al, 2005). However, according to preclinical data, biguanides can exert a direct effect in cancer cells but also an indirect effect by correcting the pro-cancerous metabolic-inflammatory aberrations that exist in diabetic patients (Foretz et al, 2014). The fact that metformin requires the presence of a membrane transporter to enter cancer cells (but not phenformin; Sogame et al, 2009) together with the observation that phenformin shows more potent anticancer effects than metformin (Yuan et al, 2013) suggest that the ideal agent to test in prospective clinical trials in patients not selected by being diabetic would be phenformin. Nonetheless, a recent phase III trial adding metformin to standard chemotherapy in pancreatic cancer did not show meaningful benefits of this intervention (Kordes et al, 2015).

In addition, studies of tumour cells indicated that our findings did not represent an autonomous response to TKIs, as none of the reprogramming events involving signalling pathways, transcriptomics, and metabolomics were observed in vitro. These findings suggest that the metabolic switch requires the interaction of several specialised cell lineages in the tumour microenvironment. We have termed this phenomenon 'metabolic synthetic lethality'. Our work considers that the tumour behaves 'as a whole' and that the phenotype is a reflection of the largest proportion of cells in the tumour (Figure 2). Obviously, our model does not imply that $100 \%$ of the cells within a tumour undergo the same type of reprogramming, but as long as the epithelial cell compartment 


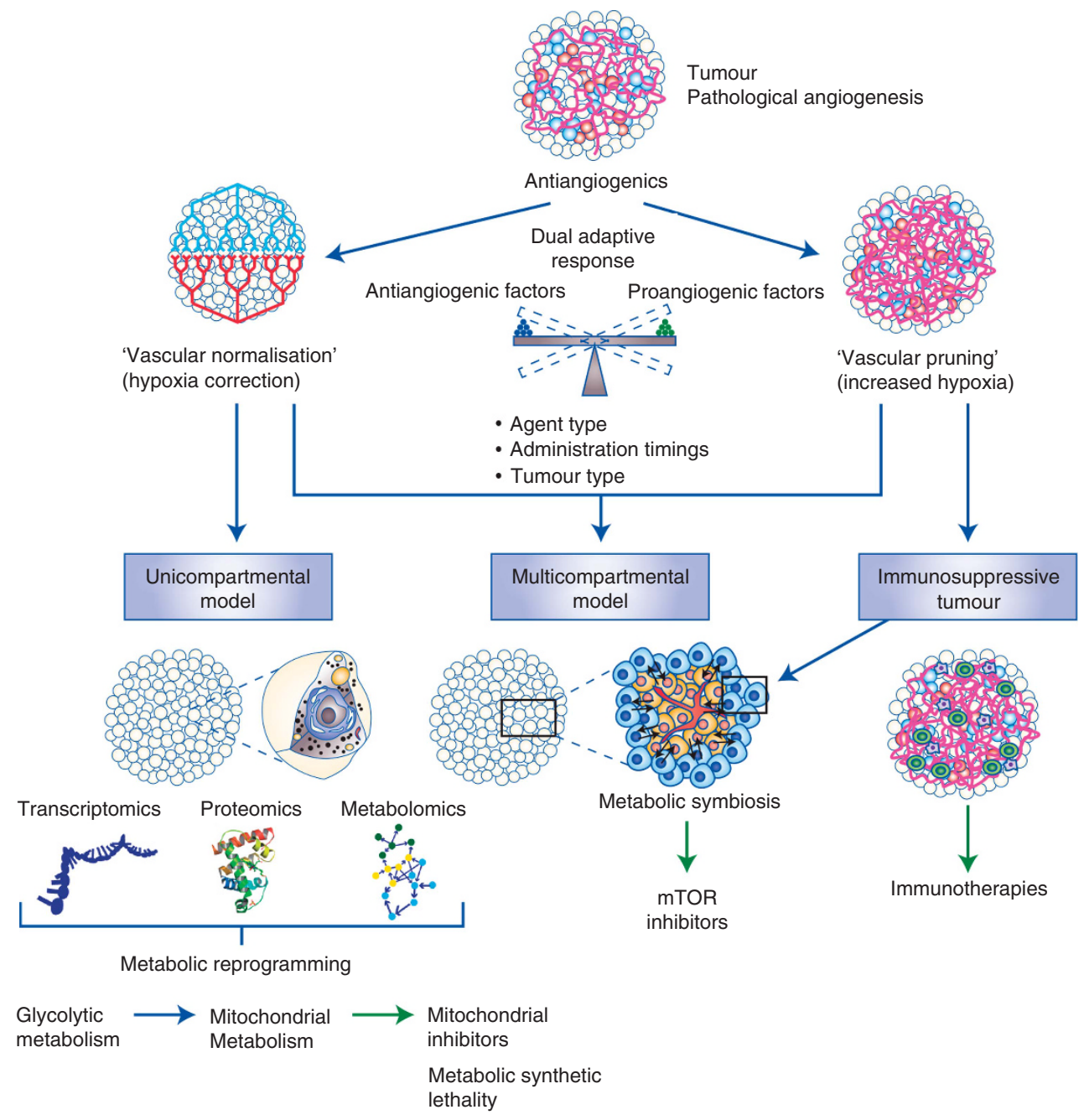

Figure 2. Dual microenviromental adaptive response against antiangiogenic therapies and its clinical implications. Tumours can exhibit a dual different adaptive response, vascular 'normalisation' (associated to hypoxia correction) or vascular pruning (associated to increased hypoxia), that would be determined by the type of antiangiogenic agent, dynamic changes in the concentrations of pro- and antiangiogenic factors, administration timing, and tumour type. Vascular normalisation corrects oxygenation leading to a metabolic switch. This metabolic reprogramming requires the interaction of several specialised cell lineages in the tumour microenvironment and it is characterised by an increased in dependence on mitochondrial metabolism. Upon this situation tumours become vulnerable to mitochondrial inhibitors inducing the phenomenon of metabolic synthetic lethality. The process may not be homogeneous: some tumour areas may experience hypoxia correction and others hypoxia increase. In this case, an alternative model proposes a metabolic compartmentalisation of tumours. In hypoxic regions cells import and metabolise glucose, whereas in normoxic regions tumour cells activate lactate catabolism leading to the upregulation of mTOR signalling. The resulting upregulation of mTOR signalling could be disrupted by the administration of mTOR inhibitors. Finally, the reprogramming may not be limited to cancer metabolism: vascular pruning and increased hypoxia as response against antiangiogenics can be associated with an immunosuppressive phenotype opening an opportunity for the novel immunotherapies.

makes up a large proportion of the volume of the tumour, the 18F-MISO-PET uptake of epithelial cells can be used to guide therapeutic decisions. However, the work of other investigators, described in the following sections, reveal the reality to be much more complex and probably difficult to fine-tune.

\section{ANTIANGIOGENIC RESISTANCE:} MULTICOMPARTMENTAL MODEL

An alternative model for understanding tumour metabolism was described by Lisanti's group as the cooperation between different tumour subpopulations, a model they named the 'reverse Warburg effect' (Pavlides et al, 2009). Basically, epithelial cancer cells induce aerobic glycolysis in neighbouring stromal fibroblasts, and the resulting energy-rich metabolites (lactate and pyruvate) are then transferred to the epithelial cancer cells, where they enter the Krebs cycle, resulting in production of high levels of ATP. Thus, the fibroblastic tumour stroma 'feeds' the cancer cells, representing a type of host-parasite relationship. The shuttle of energy-rich substrates between these cell populations occurs because cancer epithelial cells upregulate their expression of monocarboxylate transporter 1 (MCT1; a lactate importer) and induce the expression of MCT4 (a lactate exporter) in fibroblasts (WhitakerMenezes et al, 2011). Cancer cells induce transformation of normal stroma by secreting hydrogen peroxide, which triggers oxidative stress in nearby fibroblasts, resulting in reduced mitochondrial activity and increased glucose uptake (Martinez-Outschoorn et al, 2011). This metabolic compartmentalisation was shown for subcompartments of tumour epithelial cells as well. Sonveaux et al (2008) demonstrated that compartmentalisation of tumour cells was a particular form of adaptation to hypoxia. In hypoxic 
regions, cells import and metabolise glucose by glycolysis, with upregulation of the expression of the glucose importer GLUT1 and MCT4. In normoxic regions, tumour cells activate oxidative metabolism; they express the lactate importer MCT1 and avidly import lactate anions and glutamine. Therefore, a metabolic symbiosis, which can be disrupted by inhibiting one of the components such as the MCT1 transporter in the symbiotic pathways, allows the mutual survival of these two regions (Sonveaux et al, 2008). Metabolic symbiosis was recently examined in different tumour models, and found to be associated with acquired resistance to antiagiogenics (Allen et al, 2016; JimenezValerio et al, 2016; Pisarsky et al, 2016). In response to the vascular collapse and consequent hypoxia induced by antiangiogenic agents, cancer cells are compartmentalised based on their occurrence in hypoxic or normoxic regions, which depend on distance from the few remaining functional blood vessels. Under these conditions, hypoxic cancer cells induce the expression of GLUT1 and MCT4, and normoxic cells express the lactate transporter MCT1. Moreover, the metabolic pathway of lactate catabolism increases glutamine metabolism, leading to the upregulation of mammalian target of rapamycin (mTOR) signalling (Allen et al, 2016). The resulting metabolic symbiosis and upregulation of mTOR signalling in normoxic cells could be disrupted by the concomitant administration of antiangiogenic agents and rapamycin or everolimus, translated in significant antitumor effects (Allen et al, 2016; Jimenez-Valerio et al, 2016). All of these findings underscore the fact that tumour heterogeneity and plasticity are the main barriers to achieving durable responses with targeted agents used as monotherapy. The occurrence of a unicompartmental or multicompartmental response may depend on the type of agent administered and whether its effects lead to global or spatial normalisation. Investigating the reprogramming events occurring in each tumour subpopulation may help clarify the mechanisms of resistance and result in rational, tailored, synergistic regimens for those patients who develop acquired resistance against antiangiogenics agents (Figure 2).

ANTIANGIOGENIC RESISTANCE IN THE CONTEXT OF INCREASED HYPOXIA: IMMUNE REPROGRAMMING

A critical step in tumour progression is the ability of tumour cells to evade immunosurveillance by the immunocompetent host. Antiangiogenics can correct or increase hypoxia, and this can be limited to certain tumour areas or in the whole tumour volume. Regardless of the extent of the antiangiogenic-induced hypoxia, in this context the 'reprogramming' may not be limited to tumour metabolism. Emerging data indicate that tumour hypoxia is related to an immunosuppressive phenotype associated with the upregulation of the transcription factor HIF1- $\alpha$. An immunosuppressive microenvironment is promoted by HIF1- $\alpha$ by recruiting regulatory $\mathrm{T}$ cells, and increasing the expression of programmed death-ligand 1 (PD-L1) in tumour cells and myeloid-derived suppressor cells. Moreover, the growth factors and cytokines (e.g., transforming growth factor $\beta$ and VEGF) induced by hypoxia suppress the activity of T lymphocytes and inhibit the ability of dendritic cells to process tumour antigens and present them to lymphocytes (reviewed in Kumar and Gabrilovich, 2014; Palazon et al, 2014). Preclinical observations have already suggested that hypoxia correction by antiangiogenics are associated to correction of the immunosuppressive environment as well (Huang et al, 2012). To date, the published evidence about the opposite situation (antiangiogenics that increase hypoxia because of vascular pruning) is scant; our ongoing (unpublished) research suggests that immune reprogramming could be an interesting focus of research on methods that enhance the activity of immunomodulatory agents. However, the steps leading to adaptation events and their targetability remain to be elucidated.

\section{CLINICAL IMPLICATIONS}

The findings we have discussed are important because of their immediate clinical applicability. To induce the phenomenon of metabolic synthetic lethality for patients with early breast cancer, we have already launched a phase I trial that combines bevacizumab treatment with weekly doses of ME-344 started 1 week after the first dose of antiangiogenic agent. A second trial investigating the combination of phenformin with nintedanib for patients with lung or colon cancer will start at the end of the year. Finally, a third investigator-initiated trial is exploring the effects of adding anti-PD-L1 treatment to durvalumab for patients with advanced breast cancer who progressed while on bevacizumab maintenance treatment. On the basis of the recently published study investigating lenvatinib and everolimus (launched before the metabolic symbiosis hypothesis was tested at the preclinical level; Motzer et al, 2015), other trials are exploring the addition of mTOR inhibitors for patients with kidney cancer.

However, optimising the findings, that is, applying the right treatment combination (immune or metabolic modulators) at the individual level, requires the investigation of two remaining questions. First, we must confirm that the pattern of stromal adaptation can be detected in the metastatic setting. In our preoperative study, we only included patients with early breast cancer and single lesions (Quintela-Fandino et al, 2016). We cannot rule out the hypothesis that in the metastatic setting, heterogeneous adaptive patterns might be found, with some tumour lesions showing increased and others showing decreased hypoxia. Such a scenario would complicate clinical decision making. Second, although we showed how corrected or increased hypoxia correlated, respectively, with treatment efficacy or lack of it, we have not yet demonstrated in the clinical setting that the addition of a metabolic inhibitor or an immunomodulator is associated with enhanced therapeutic effects. In addition, a quantitative assessment of peripheral blood specimens would be preferable to imaging tests for guiding therapeutic decisions. Ongoing research for peripheral blood biomarkers is focused on hydroxylated proteins.

Angiogenesis is a complex process, and understanding the mechanisms of resistance in order to optimise therapy seems to depend both on the mechanism of action of the specific agent under study and the tumour type, which requires assessment of each individual patient. Novel lines of research on resistance to antioangiogenic agents extend beyond the study of the implications of several signalling axes and point towards both the cooperation between several cell lineages and metabolic reprogramming. Personalised decision making may be possible by incorporating novel hypoxia/normoxia biomarkers with recent discoveries on dual adaptive patterns of resistance and may prolong the time that metastatic patients benefit from a class of drugs for which precision medicine has been elusive.

\section{CONFLICT OF INTEREST}

The authors declare no conflict of interest.

\section{REFERENCES}

Allen E, Mieville P, Warren CM, Saghafinia S, Li L, Peng MW, Hanahan D (2016) Metabolic symbiosis enables adaptive resistance to antiangiogenic therapy that is dependent on mTOR signaling. Cell Rep 15(6): 1144-1160. 
Choueiri TK, Escudier B, Powles T, Mainwaring PN, Rini BI, Donskov F, Hammers H, Hutson TE, Lee JL, Peltola K, Roth BJ, Bjarnason GA, Geczi L, Keam B, Maroto P, Heng DY, Schmidinger M, Kantoff PW, Borgman-Hagey A, Hessel C, Scheffold C, Schwab GM, Tannir NM, Motzer RJ, Investigators M (2015) Cabozantinib versus everolimus in advanced renal-cell carcinoma. $N$ Engl J Med 373(19): 1814-1823.

Cooke VG, LeBleu VS, Keskin D, Khan Z, O'Connell JT, Teng Y, Duncan MB, Xie L, Maeda G, Vong S, Sugimoto H, Rocha RM, Damascena A, Brentani RR, Kalluri R (2012) Pericyte depletion results in hypoxiaassociated epithelial-to-mesenchymal transition and metastasis mediated by met signaling pathway. Cancer Cell 21(1): 66-81.

Evans JM, Donnelly LA, Emslie-Smith AM, Alessi DR, Morris AD (2005) Metformin and reduced risk of cancer in diabetic patients. BMJ 330(7503): 1304-1305.

Folkman J (1971) Tumor angiogenesis: therapeutic implications. N Engl J Med 285(21): 1182-1186.

Folkman J (1990) What is the evidence that tumors are angiogenesis dependent? J Natl Cancer Inst 82(1): 4-6.

Foretz M, Guigas B, Bertrand L, Pollak M, Viollet B (2014) Metformin: from mechanisms of action to therapies. Cell Metab 20(6): 953-966.

Hernandez-Agudo E, Mondejar T, Soto-Montenegro ML, Megias D, Mouron S, Sanchez J, Hidalgo M, Lopez-Casas PP, Mulero F, Desco M, Quintela-Fandino M (2016) Monitoring vascular normalization induced by antiangiogenic treatment with (18)F-fluoromisonidazole-PET. Mol Oncol 10(5): 704-718.

Huang Y, Yuan J, Righi E, Kamoun WS, Ancukiewicz M, Nezivar J, Santosuosso M, Martin JD, Martin MR, Vianello F, Leblanc P, Munn LL, Huang P, Duda DG, Fukumura D, Jain RK, Poznansky MC (2012) Vascular normalizing doses of antiangiogenic treatment reprogram the immunosuppressive tumor microenvironment and enhance immunotherapy. Proc Natl Acad Sci USA 109(43): 17561-17566.

Hurwitz H, Fehrenbacher L, Novotny W, Cartwright T, Hainsworth J, Heim W, Berlin J, Baron A, Griffing S, Holmgren E, Ferrara N, Fyfe G, Rogers B, Ross R, Kabbinavar F (2004) Bevacizumab plus irinotecan, fluorouracil, and leucovorin for metastatic colorectal cancer. $N$ Engl $J$ Med 350(23): 2335-2342.

Jain RK (2013) Normalizing tumor microenvironment to treat cancer: bench to bedside to biomarkers. J Clin Oncol 31(17): 2205-2218.

Jimenez-Valerio G, Martinez-Lozano M, Bassani N, Vidal A, Ochoa-de-Olza M, Suarez C, Garcia-Del-Muro X, Carles J, Vinals F, Graupera M, Indraccolo S, Casanovas O (2016) Resistance to antiangiogenic therapies by metabolic symbiosis in renal cell carcinoma PDX models and patients. Cell Rep 15(6): 1134-1143.

Kordes S, Pollak MN, Zwinderman AH, Mathot RA, Weterman MJ, Beeker A, Punt CJ, Richel DJ, Wilmink JW (2015) Metformin in patients with advanced pancreatic cancer: a double-blind, randomised, placebocontrolled phase 2 trial. Lancet Oncol 16(7): 839-847.

Kumar SM, Yu H, Edwards R, Chen L, Kazianis S, Brafford P, Acs G, Herlyn M, Xu X (2007) Mutant V600E BRAF increases hypoxia inducible factor-1alpha expression in melanoma. Cancer Res 67(7): 3177-3184.

Kumar V, Gabrilovich DI (2014) Hypoxia-inducible factors in regulation of immune responses in tumour microenvironment. Immunology 143(4): 512-519.

Liu LP, Ho RL, Chen GG, Lai PB (2012) Sorafenib inhibits hypoxia-inducible factor-1alpha synthesis: implications for antiangiogenic activity in hepatocellular carcinoma. Clin Cancer Res 18(20): 5662-5671.

Lu KV, Chang JP, Parachoniak CA, Pandika MM, Aghi MK, Meyronet D, Isachenko N, Fouse SD, Phillips JJ, Cheresh DA, Park M, Bergers G (2012) VEGF inhibits tumor cell invasion and mesenchymal transition through a MET/VEGFR2 complex. Cancer Cell 22(1): 21-35.

Lunt SY, Vander Heiden MG (2011) Aerobic glycolysis: meeting the metabolic requirements of cell proliferation. Annu Rev Cell Dev Biol 27: 441-464.

Martinez-Outschoorn UE, Lin Z, Trimmer C, Flomenberg N, Wang C, Pavlides S, Pestell RG, Howell A, Sotgia F, Lisanti MP (2011) Cancer cells metabolically 'fertilize' the tumor microenvironment with hydrogen peroxide, driving the Warburg effect: implications for PET imaging of human tumors. Cell Cycle 10(15): 2504-2520.

Matsumoto S, Batra S, Saito K, Yasui H, Choudhuri R, Gadisetti C, Subramanian S, Devasahayam N, Munasinghe JP, Mitchell JB, Krishna MC (2011) Antiangiogenic agent sunitinib transiently increases tumor oxygenation and suppresses cycling hypoxia. Cancer Res 71(20): 6350-6359.
Monk BJ, Poveda A, Vergote I, Raspagliesi F, Fujiwara K, Bae DS, Oaknin A, Ray-Coquard I, Provencher DM, Karlan BY, Lhomme C, Richardson G, Rincon DG, Coleman RL, Herzog TJ, Marth C, Brize A, Fabbro M, Redondo A, Bamias A, Tassoudji M, Navale L, Warner DJ, Oza AM (2014) Anti-angiopoietin therapy with trebananib for recurrent ovarian cancer (TRINOVA-1): a randomised, multicentre, double-blind, placebo-controlled phase 3 trial. Lancet Oncol 15(8): 799-808.

Motzer RJ, Hutson TE, Glen H, Michaelson MD, Molina A, Eisen T, Jassem J, Zolnierek J, Maroto JP, Mellado B, Melichar B, Tomasek J, Kremer A, Kim HJ, Wood K, Dutcus C, Larkin J (2015) Lenvatinib, everolimus, and the combination in patients with metastatic renal cell carcinoma: a randomised, phase 2, open-label, multicentre trial. Lancet Oncol 16(15): 1473-1482.

Murphy DA, Makonnen S, Lassoued W, Feldman MD, Carter C, Lee WM (2006) Inhibition of tumor endothelial ERK activation, angiogenesis, and tumor growth by sorafenib (BAY43-9006). Am J Pathol 169(5): $1875-1885$.

Navarro P, Bueno MJ, Zagorac I, Mondejar T, Sanchez J, Mouron S, Munoz J, Gomez-Lopez G, Jimenez-Renard V, Mulero F, Chandel NS, QuintelaFandino M (2016) Targeting tumor mitochondrial metabolism overcomes resistance to antiangiogenics. Cell Rep 15(12): 2705-2718.

Oliner J, Min H, Leal J, Yu D, Rao S, You E, Tang X, Kim H, Meyer S, Han SJ, Hawkins N, Rosenfeld R, Davy E, Graham K, Jacobsen F, Stevenson S, Ho J, Chen Q, Hartmann T, Michaels M, Kelley M, Li L, Sitney K, Martin F, Sun JR, Zhang N, Lu J, Estrada J, Kumar R, Coxon A, Kaufman S, Pretorius J, Scully S, Cattley R, Payton M, Coats S, Nguyen L, Desilva B, Ndifor A, Hayward I, Radinsky R, Boone T, Kendall R (2004) Suppression of angiogenesis and tumor growth by selective inhibition of angiopoietin-2. Cancer Cell 6(5): 507-516.

Palazon A, Goldrath AW, Nizet V, Johnson RS (2014) HIF transcription factors, inflammation, and immunity. Immunity 41(4): 518-528.

Pavlides S, Whitaker-Menezes D, Castello-Cros R, Flomenberg N, Witkiewicz AK, Frank PG, Casimiro MC, Wang C, Fortina P, Addya S, Pestell RG, Martinez-Outschoorn UE, Sotgia F, Lisanti MP (2009) The reverse Warburg effect: aerobic glycolysis in cancer associated fibroblasts and the tumor stroma. Cell Cycle 8(23): 3984-4001.

Pepper MS, Ferrara N, Orci L, Montesano R (1992) Potent synergism between vascular endothelial growth factor and basic fibroblast growth factor in the induction of angiogenesis in vitro. Biochem Biophys Res Commun 189(2): 824-831.

Pisarsky L, Bill R, Fagiani E, Dimeloe S, Goosen RW, Hagmann J, Hess C, Christofori G (2016) Targeting metabolic symbiosis to overcome resistance to anti-angiogenic therapy. Cell Rep 15(6): 1161-1174.

Quintela-Fandino M, Lluch A, Manso LM, Calvo I, Cortes J, Garcia-Saenz JA, Gil JM, Martinez-Janez N, Gonzalez-Martin A, Adrover E, De Andres R, Vinas G, Llombart Cussac A, Alba E, Guerra J, Bermejo B, Zamora E, Moreno-Anton F, Pernas-Simon S, Carrato A, Lopez A, Escudero MJ, Campo R, Carrasco EM, Palacios J, Mulero F, Colomer R (2016) 18F-fluoromisonidazole PET and activity of neoadjuvant nintedanib in early HER2-negative breast cancer: a window-of-opportunity randomized trial. Clin Cancer Res; e-pub ahead of print 1 September 2016; doi:10.1158/ 1078-0432.CCR-16-0738.

Sennino B, McDonald DM (2012) Controlling escape from angiogenesis inhibitors. Nat Rev Cancer 12(10): 699-709.

Sogame Y, Kitamura A, Yabuki M, Komuro S (2009) A comparison of uptake of metformin and phenformin mediated by hOCT1 in human hepatocytes. Biopharm Drug Dispos 30(8): 476-484.

Sonveaux P, Vegran F, Schroeder T, Wergin MC, Verrax J, Rabbani ZN, De Saedeleer CJ, Kennedy KM, Diepart C, Jordan BF, Kelley MJ, Gallez B, Wahl ML, Feron O, Dewhirst MW (2008) Targeting lactate-fueled respiration selectively kills hypoxic tumor cells in mice. J Clin Invest 118(12): 3930-3942.

Van der Veldt AA, Lubberink M, Bahce I, Walraven M, de Boer MP, Greuter HN, Hendrikse NH, Eriksson J, Windhorst AD, Postmus PE, Verheul HM, Serne EH, Lammertsma AA, Smit EF (2012) Rapid decrease in delivery of chemotherapy to tumors after anti-VEGF therapy: implications for scheduling of anti-angiogenic drugs. Cancer Cell 21(1): 82-91.

Whitaker-Menezes D, Martinez-Outschoorn UE, Lin Z, Ertel A, Flomenberg N, Witkiewicz AK, Birbe RC, Howell A, Pavlides S, Gandara R, Pestell RG, Sotgia F, Philp NJ, Lisanti MP (2011) Evidence for a stromal-epithelial 'lactate shuttle' in human tumors: MCT4 is a 
marker of oxidative stress in cancer-associated fibroblasts. Cell Cycle 10(11): 1772-1783.

Yuan P, Ito K, Perez-Lorenzo R, Del Guzzo C, Lee JH, Shen CH, Bosenberg MW, McMahon M, Cantley LC, Zheng B (2013) Phenformin enhances the therapeutic benefit of BRAF(V600E) inhibition in melanoma. Proc Natl Acad Sci USA 110(45): 18226-18231. (c) (1)(2) (2) This work is licensed under the Creative Commons c) Attribution-Non-Commercial-Share Alike 4.0 International License. To view a copy of this license, visit http:// creativecommons.org/licenses/by-nc-sa/4.0/

(C) The Author(s) named above 2017 